\title{
Exosomal miR-638 Inhibits Hepatocellular Carcinoma Progression by Targeting SPI
}

\section{Jing Yang \\ Bo $\mathrm{Li}^{1}$ \\ Shuo Zhao ${ }^{2}$ \\ Hongyu Du' \\ Yaming $\mathrm{Du}$ (iD) \\ 'Department of Pathology, Jinzhou Medical University, Jinzhou, Liaoning, People's Republic of China; ${ }^{2}$ Department of Nursing, The First Affiliated Hospital of Jinzhou Medical University, Jinzhou, Liaoning, People's Republic of China; ${ }^{3}$ Department of Vascular Surgery, The First Affiliated Hospital of Jinzhou Medical University, Jinzhou, Liaoning, People's Republic of China}

\author{
This article was published in the following Dove Press journal: \\ OncoTargets and Therapy
}

Purpose: Exosomal microRNAs (miRNAs) play essent roles in the evelopment of hepatocellular carcinoma (HCC). Nevertheless, the re and h hanism of xosomal miR638 in HCC development remain largely unknow

Methods: Exosomes were isolated and confirn via tra nission elctron microscopy and western blot. The abundances of miR-638 spee protein SP1) were measured via quantitative reverse transcription polym se chain re. ion western blot. Cell proliferation was investigated by Cell Count $\mathrm{K}_{\mathrm{H}}$ colony fon assay, apoptosis, cell cycle distribution and related protein expression. C migration and invasion were detected via transwell assay and western ot. 0 -culture exp nent was performed to assess exosome transfer from HCC cells t endothelial cells. The target correlation between miR-638 and SP1 was analyzed via dua uciferase re ter and RNA immunoprecipitation assays. The subcutaneous xenograft expc nent was nducted to test the function of miR-638 in vivo. Results: The m level dec. in exosomes from serum or HCC cell medium. miR638 overexpressic repre HCC cell proliferation by decreasing viability and colony formationand indu apoptosis and cell cycle arrest at G1 phase, and decreased abilities of $\mathrm{p}$, ration and in ion. Exosomal miR-638 from HCC cells could transfer to human silical y 1 endoth al cells (HUVECs) and suppress HUVEC proliferation, migration anc Vo on. SPI was a target of miR-638 and overexpression of SP1 reversed the effect of miR-6. on HCC cells. Overexpression of miR-638 reduced xenograft tumor growth via decreasing $\mathrm{P} 1$.

onclusion: Exosomal miR-638 inhibited HCC tumorigenesis by targeting SP1. This study in ted the potential clinical implications of miR-638 in HCC.

Keywords: hepatocellular carcinoma, exosome, miR-638, SP1

\section{Introduction}

Liver cancer is a public malignancy with the second leading cause of cancer-related deaths, and hepatocellular carcinoma (HCC) represents more than $90 \%$ of cases. ${ }^{1}$ In recent years, new hope has been brought for HCC patients with the progress of treatment options. ${ }^{2}$ Nevertheless, the outcome of patients remains unsatisfactory. Therefore, it is urgent to find a new strategy for the treatment of HCC.

Exosomes are a class of extracellular vesicles that take part in intercellular communication in HCC. ${ }^{3}$ Exosomes play important roles in the tumorigenesis, diagnosis and treatment of $\mathrm{HCC}$ by transmitting nucleic acids, proteins or lipids. ${ }^{4}$ Noncoding RNAs are enriched in exosomes and exosomal noncoding RNAs have essential roles in HCC development. ${ }^{5}$ MicroRNAs (miRNAs) are small noncoding

Correspondence: Yaming Du Email yangjing963I@163.com RNAs which could be transferred by exosomes to participate in the progression and 
therapeutics of HCC. ${ }^{6}$ Previous studies suggest that miR638 has an important clinical significance in HCC development. ${ }^{7,8}$ Moreover, knockdown of miR-638 could promote HCC development by increasing cell growth, angiogenesis, migration and invasion. ${ }^{9,10}$ In addition, miR-638 could be enriched in exosomes, and exosomal miR-638 plays a key role in human cancers. ${ }^{11,12}$ More importantly, serum exosomal miR-638 has an important prognostic role in HCC. ${ }^{13}$ However, the mechanism of exosomal miR-638 in HCC development remains largely unclear.

miRNAs exhibit their roles in $\mathrm{HCC}$ by regulating gene expression and translation. ${ }^{14}$ Specificity protein 1 (SP1) is overexpressed and plays an oncogenic role in many cancers by regulating cell proliferation, differentiation, apoptosis and angiogenesis. ${ }^{15}$ Accruing evidence indicates that SP1 is associated with cell proliferation, migration, invasion and angiogenesis in HCC. ${ }^{16-18}$ Moreover, starBase online predicts that SP1 might act as a target of miR-638. Hence, we hypothesized that exosomal miR-638 might target SP1 to mediate HCC progression. In the present research, we measured the exosomal miR-638 level, and investigated the effect of miR-638 on HCC development. Moreover, we analyzed the target association between miR-638 and SF
Table I The Relationship Between miR-638 Expression and Clinicopathological Features in HCC $(n=42)$

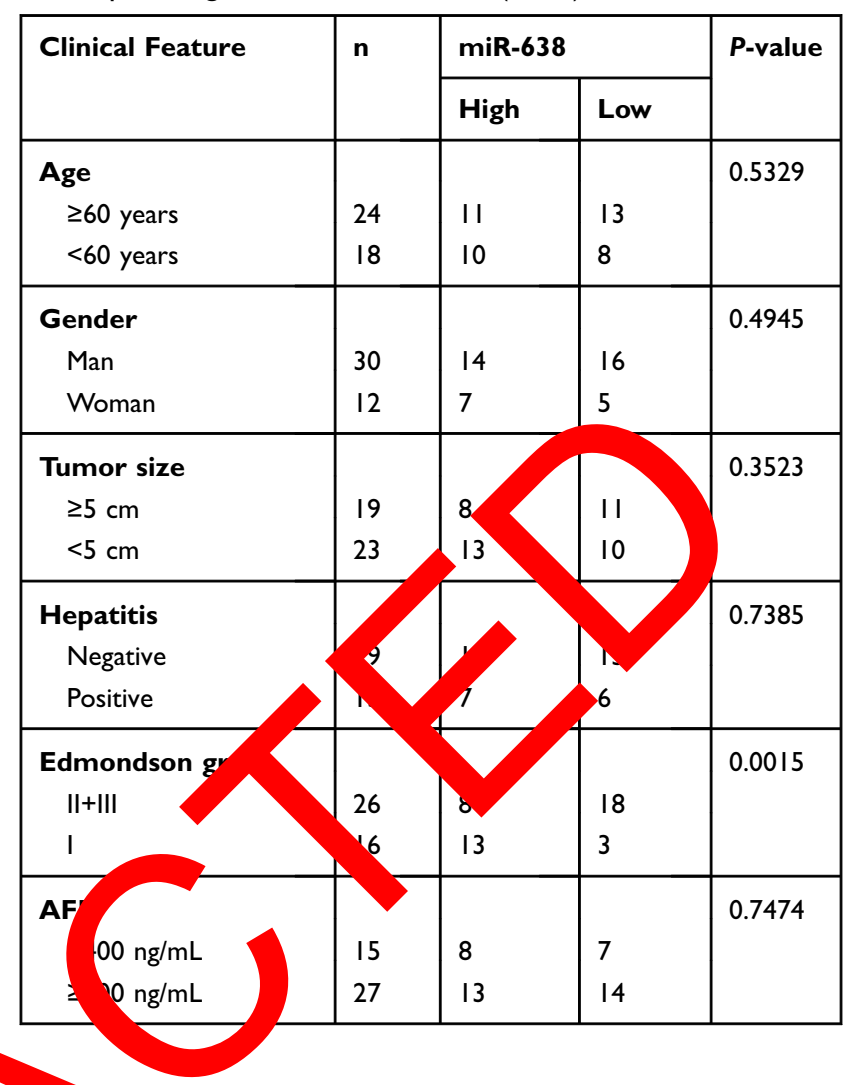

\section{Patients and Methods} Patient Tissues and Serum

Forty-two HCC patients and 20 nor

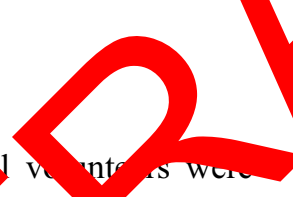
recruited from Jinzhou Medical Un crsity. Tho eripheral blood samples were harvested a trifuged for erum collection. The cancer and pa -tumor tiss s were obtained from HCC patients. The rents did not rea any other therapy prior to samp collect 1 . Written informed consent was obtained from a ojects. $T$ patients' features are displayed Tay 1 . S st $s^{t} y$ was carried out in accordance th the uidelines Declaration of Helsinki" and under the valorm thics Committee of Jinzhou Medical Univers

\section{Cell Culture and Treatment}

Human umbilical vein endothelial cells (HUVECs), HCC cell lines (MHCC97-H, HCCLM3 and Huh7) and normal human liver cell line THLE-2 were provided via Bena Culture Collection (Beijing, China). DMEM (Sigma, St. Louis, MO, USA) with $10 \%$ fetal bovine serum (Biosun, Shanghai, China) as well as 1\% penicillin-streptomycin (Sigma) was applied to cell culture. The cells were

\section{Exosome Purification and Validation}

The exosomes were purified from serum or cell medium using the Exoquick exosome extraction kit (SBI System Biosciences, Mountain View, CA, USA) according to the instructions. ${ }^{19}$ The exosomes were validated by transmission electron microscopy (TEM) (Hitachi, Tokyo, Japan) and expression of exosomal markers. ${ }^{20,21}$

\section{Quantitative Reverse Transcription Polymerase Chain Reaction (qRT-PCR)}

Exosomal RNA was isolated using the Exosome Purification and RNA Isolation kit (Amyjet Scientific, Wuhan, China), and RNA from tissues or cells was extracted using TRIzol (Solarbio, Beijing, China). The cDNA was generated using the specific reverse transcription kit (Thermo Fisher, Wilmington, DE, USA). The cDNA was mixed with SYBR Green (Vazyme, Nanjing, China) and specific primers, and then applied to qRT-PCR. The primers were synthesized from Genscript (Nanjing, 
China) and shown as: SP1 (sense, 5'-CGCCCTCTGAC CAAGATCACT-3'; antisense, 5'-GGGAGTTGTTGCTGT TCTCATTGG-3'), miR-638 (sense, 5'-GAGAGGATCCT GCCGCAGATCGCTG-3'; antisense, GAGTAAGCTTCA GGGAGTCCTCTGCC), U6 (sense, 5'-ACGCAAATTCG TGAAGCGTT-3'; antisense, AACGCTTCACGAATTT GCGT) and GAPDH (sense, 5'-GACAGTCAGCCGCAT CTTCT-3'; antisense, 5'-GCGCCCAATACGACCAAATC $\left.-3^{\prime}\right)$. The relative expression of SP1 or miR-638 was calculated with GAPDH or U6 as a reference according to the delta-delta cycle threshold method. ${ }^{22}$

\section{Cell Transfection}

SP1 overexpression vector was generated using pcDNA3.1 (Thermo Fisher). The pcDNA3.1 vector was exploited as a negative control (vector). miR-638 mimic (5'-AGGGA UCGCGGGCGGGUGGCGGCCU-3') and mimic negative control (NC, 5'-UUCUCCGAACGUGUCACGUTT-3') were generated via Ribobio (Guangzhou, China). Huh7 cells were transfected with 50 or $100 \mathrm{nM}$ oligonucleotides or $600 \mathrm{ng}$ vectors via Lipofectamine 3000 reagent (Thermo Fisher) for $24 \mathrm{~h}$. The non-transfected cells were regarded as the control group.

\section{Co-Culture of HUVECs and Huh7 Ce "s} Huh7 cells were transfected with miR-638 and then the exosomes were isolated, (miR-638) or Huh7-exo(NC) group, date that miR-638 was transferred no ed a as uh7-e exo $\mathrm{Huh} 7 \mathrm{cells}$ transfected with miR-638 vic were treated by SW4869, and then exoso res w isolated, named as Huh7-exo(miR-638)-G 4869 group. he exosomes were co-cultured with Hy ECs for $48 \mathrm{~h}$.

\section{Cell Counting $-8(C, \mathrm{C}-8)$}

The viab' $y$ of $\mathrm{h}$ h7 cen aUVECs was examined via CCK- ssay. 5 alls were added into 96-well plates and incub for 24,48 and $72 \mathrm{~h}$. Then, $10 \mu \mathrm{L}$ CCK-8 reagent (Ape. $\mathrm{Q}$, Houston, TX, USA) was introduced and the cells were maintained for another $4 \mathrm{~h}$. The optical density (OD) value was measured through a microplate reader (Bio-Gene Technology, Guangzhou, China). The detection wavelength was $450 \mathrm{~nm}$.

\section{Colony Formation Assay}

Huh7 cells or HUVECs (500 cells/well) were placed into 6 -well plates and cultured for 2 weeks. The clones were dyed with $0.5 \%$ crystal violet (Beyotime, Shanghai,
China), and then observed under a microscope (Agilent, Chengdu, China).

\section{Flow Cytometry}

Cell apoptosis and cycle distribution were analyzed via flow cytometry. For cell apoptosis assay, Huh7 cells or HUVECs $\left(2 \times 10^{5}\right.$ cells/well $)$ were placed into 12-well plates, and cultured for $72 \mathrm{~h}$. Next, cells were incubated in the binding buffer and interacted with Annexin V-FITC and PI (Solarbio) in the dark. The apoptotic cells were analyzed using a flow cytometer Biosciences, San Jose, CA, USA).

For the cell cycle assay, $10^{5}$ cells $\mathrm{w}$ e placed into 6 -well plates, and culty a for $\lambda_{2}$ Next, lls were fixed with $70 \%$ ethanol then reated of $\mu \mathrm{g} / \mathrm{mL}$ PI and RNase A for $30 \mathrm{~m}$ The distribution was analyzed with a flow

\section{Transwell, alysis}

Th Grated and vvasive abilities of Huh7 cells or UVECs were analyzed via 24-well transwell chambers Costar, Cori ig, NY, USA). The transwell invasion assay conduc d using chambers precoated with Matrigel, whin iranswell migration assay was conducted using

without Matrigel. $1 \times 10^{5}$ cells were resuspended in medium without serum and added into the upper chambers. The medium containing 10\% serum was injected into the lower chambers. After $24 \mathrm{~h}$, cells on the low chambers were incubated with $0.5 \%$ crystal violet. The number of migrated or invasive cells was counted under a microscope (Agilent) with three random fields.

\section{Western Blot}

The protein was isolated with RIPA buffer (Beyotime) containing $1 \mathrm{mM}$ PMSF (Beyotime), and quantified using a protein quantification kit (Abbkine, Redlands, CA, USA). $20 \mu \mathrm{g}$ samples were separated via sodium dodecyl sulfatepolyacrylamide gel electrophoresis and transferred onto polyvinylidene fluoride membranes (Solarbio). Following blocking in 5\% nonfat milk, the membranes were interacted with specific primary antibodies and secondary antibody. The antibodies were shown as: anti-TSG101 (ab30871, 1:500 dilution; Abcam, Cambridge, MA, USA), anti-CD63 (ab216130, 1:2000 dilution; Abcam), anti-HSP70 (ab31010, 1:1000 dilution; Abcam), anti-CD81 (ab109201, 1:5000 dilution; Abcam), anti-caspase 3 (total caspase 3 and Cleaved caspase 3) (ab184787, 1:2000 dilution; Abcam), anti-PCNA (ab152112, 1:2000 dilution; Abcam), anti-Cyclin D1 (ab226977, 1:2000 
dilution; Abcam), anti-MMP9 (ab73734, 1:1000 dilution; Abcam), anti-SP1 (ab227383, 1:500 dilution; Abcam) and anti-GAPDH (ab9485, 1:2000 dilution; Abcam), as well as horseradish peroxidase-conjugated IgG (ab205718, 1:20,000 dilution; Abcam). GAPDH was used as a reference. Next, the bands were visualized using the BeyoECL Plus kit (Beyotime). The relative protein level was normalized to the control group.

\section{Dual-Luciferase Reporter Analysis and RNA Immunoprecipitation (RIP)}

The complementary sequence of miR-638 and SP1 was predicted using starBase (http://starbase.sysu.edu.cn/ index.php). The 3'UTR sequence of SP1 containing miR638 binding sites was inserted into the luciferase reporter vector psiCHECK-2 (Promega, Madison, WI, USA) to synthesize the wild-type luciferase constructs SP1-wt. The mutant-type constructs were generated via mutating the binding sites, named as SP1-mut. Huh7 cells were cotransfected with $600 \mathrm{ng}$ constructs and miR-638 mimic or $\mathrm{NC}$ for $24 \mathrm{~h}$. Subsequently, luciferase activity was determined with a dual-luciferase assay kit (Promega).

For RIP assay, the RNA-Binding Prote Immunoprecipitation kit (Sigma) was used. Huh7 cel transfected with miR-638 mimic or NC were lysed, and lysates were incubated with magnetic beads salo with Ago2 antibody. IgG antibody was used as $r$ sative $c$ itrol. SP1 mRNA was extracted from beads qRT-PCR.

\section{Xenograft Experime $\pi$}

Male BALB/c nude mic 5 weels old) wereprovided via Beijing Laboratory A. nal $C$ iter (Beijing, China) and acclimatized for 1 reek. 77 cell $2 \times 10^{6}$ cells $/$ mouse) were subcuta ously hocula mice. After 9 days, mice with forme unors were divided into two groups $(\mathrm{n}=5$ per grou $\mathrm{nM}$ miR-638 agomir (miR400033084-5, Ribobio) o. $\mathrm{NC}$ was injected into the sites of tumors every other vay for 4 times. Tumor volume was measured before the injection of agomir. At 3 days after the last injection, animals were killed via cervical dislocation under anesthesia using isoflurane. Tumor tissues were collected and the weight was detected. Furthermore, the levels of miR-638, SP1, PCNA, total caspase 3 and Cleaved caspase 3 were detected in tumor tissues. The animal procedures were performed in accordance with the Guidelines for Care and Use of Laboratory Animals of "National Institutes of Health" and approved via the Institutional Animal Care and Use Committee of Jinzhou Medical University.

\section{Statistical Analysis}

All experiments were carried out 3 times. Statistical analysis was conducted using GraphPad Prism 7 (GraphPad, La Jolla, CA, USA). The data were normally distributed and shown as mean \pm SD. The linear correlation of miR- 638 and SP1 expression was analyzed via Pearson correlation analysis. The difference between group compared via Student's $t$-test or ANOVA follow via Tuk 's test. The difference was statistically signifh when $P<1 \quad 05$.

\section{Results}

miR-638 Level is Jer eased jn HCC

\section{Exosomes}

To identify ex mal miR $s$ in HCC, the exosomes were purified from um of $\mathrm{HCC}$ patients or normal subje s. As shown in sure $1 \mathrm{~A}$ and $\mathrm{B}$, the exosomes wer secreted $w$ hout an obvious difference in serum of the $\mathrm{o}$ groups, $a d$ the diameters of exosomes from HCC serum re m aly between 80 and $120 \mathrm{~nm}$. Meanwhile, a exosomes were also confirmed by the presence of somuspecific markers (TSG101, CD63, HSP70 and CD81) (Figure 1C). Moreover, the miR-638 level was gnificantly decreased in HCC serum-derived exosomes in comparison to the normal group (Figure 1D). In addition, the miR-638 level was lower in HCC tissues than para-tumor samples (Figure 1E). Besides, the abundance of miR-638 was also detected in HCC cells or mediumderived exosomes. Results exhibited that the miR-638 level was remarkably reduced in HCC cells or exosomes (Figure 1F and G). These data indicated that a low level of exosomal miR-638 was displayed in HCC serum and cell medium. Furthermore, low expression of miR-638 was associated with Edmondson grade, but not with age, gender, tumor size, hepatitis and AFP level (Table 1).

\section{Up-Regulation of miR-638 Suppresses Proliferation, Migration and Invasion of HCC Cells}

To explore the role of miR-638 in HCC cells, Huh7 cells with the relative lowest abundance of miR-638 were chosen for further experiments, and cells were transfected with 50 or $100 \mathrm{nM}$ miR-638 mimic or NC. As displayed in Figure 2A and $\mathrm{B}$, cell viability and colony formation were evidently 
A

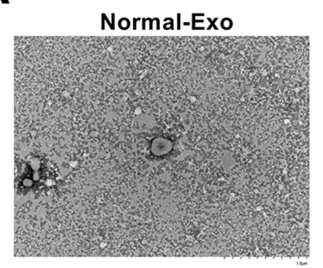

D

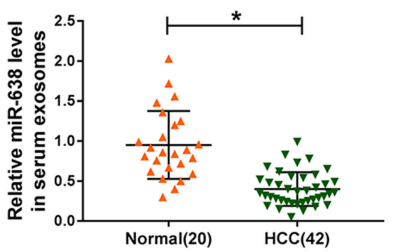

E

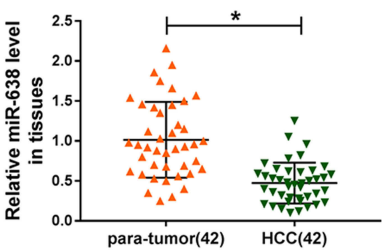

B

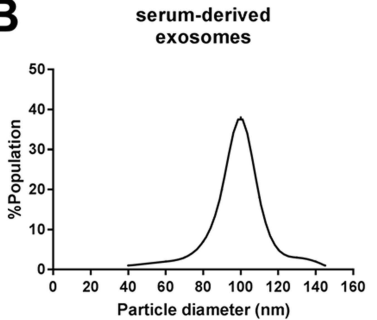

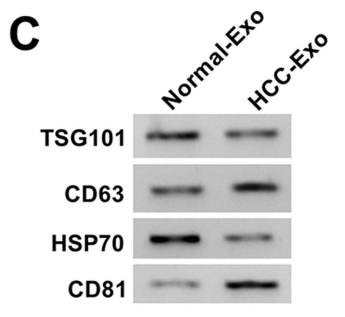

$\mathbf{F}$
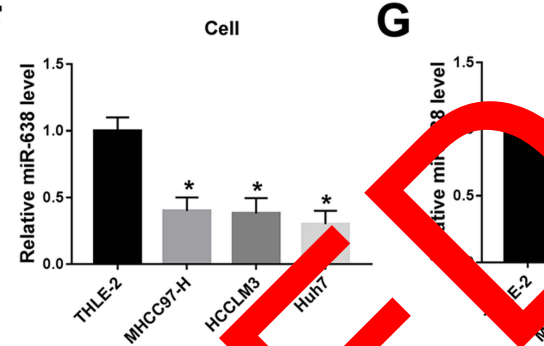

G

Exosomes

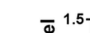

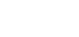

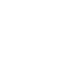

ed via 1

Figure I The level of miR-638 in HCC. (A) Representative graphs of HCC-Exo and Normal-Exo were (C) The protein levels of TSGI0I, CD63, HSP70 and CD8I were detected in HCC-Exo and Normal-Ex
exosomes from HCC patients and normal subjects via qRT-PCR. (E) miR-638 level was detected in $\mathrm{HCC}$ and normal liver cells or exosome. ${ }^{*} P<0.05$.

decreased via transfection of miR-638 mimic in a concentration-dependent manner. Furthermore, flow cytometry analysis showed that transfection of different concentrations of miR-638 mimic induced cell apoptosis a cycle arrest at G1 phase (Figure 2C and D). In additio the migrated and invasive abilities of Huh7 cellswere rem ably inhibited via different concentrations miR- $8 \mathrm{~min}$ (Figure 2E and F). Besides, the relat protein wnression was measured, such as PCNA (p motin oliferation), Cyclin D1 (regulating cell cy from G1 S phase), ${ }^{24}$ Cleaved caspase 3 (promotir, apop, is) ${ }^{25}$ and MuMP9 (promoting migration and asion). ${ }^{26}$ Res s showed that elevation of Cleaved case 3 a reduction of PCNA, Cyclin D1 and MMP9 wo ind ed by transfection of miR-638 mimic in $\mathrm{H}^{2}$ ells vure 26 These results suggested that mir 038 erexpro using miR-638 mimic repress $\mathrm{HCC}$ -

\section{Exosomal R-638 Inhibits Endothelial Function}

Endothelial function is responsible for tumor angiogenesis in HCC. To assess the influence of exosomal miR-638 on endothelial function, Huh7 cells were transfected with miR-638 mimic or NC, and exosomes in medium were collected and used for co-culture with HUVECs. As displayed in Figure 3A-D, Huh7 cell-derived exosomal miR638 obviously repressed HUVEC proliferation via creasing viability and colony formation and promoting poptosis an affecting cell cycle distribution, while these ents were orogated via GW4869, an inhibitor of exoson - ion. After the treatment of GW4869, the nearance of exosome and miR-638 expression were reduced (Supplementary Figure 1A and $\underline{B}$ ). Moreover, Huh7 cell-derived exosomal miR-638 significantly inhibited HUVEC migration and invasion, which was reversed by GW4869 (Figure 3E and F). Besides, Huh7 cell-derived exosomal miR-638 evidently enhanced the level of Cleaved caspase 3 and declined the protein levels of PCNA, Cyclin D1 and MMP9 in HUVECs, and treatment of GW4869 abolished this effect (Figure 3G). These findings indicated that exosomal miR-638 might inhibit HCC angiogenesis by regulating endothelial function.

\section{SPI is a Target of miR-638 in HCC Cells}

To explore the mechanism of miR-638 in HCC, the targets of miR-638 were predicted via starBase. Among these targets, SP1 expression was enhanced at mRNA and protein levels and negatively correlated with miR-638 level in HCC tissues (Figure 4A-C). Moreover, SP1 protein expression was evidently elevated in HCC cells (Figure 4D). To further confirm the target association between miR-638 and SP1, SP1-wt and SP1-mut were constructed (Figure 4E), and used for dual-luciferase reporter analysis. Results displayed that addition of miR-638 induced a $76 \%$ reduction in luciferase activity of SP1-wt, but its effect was abolished via mutating 


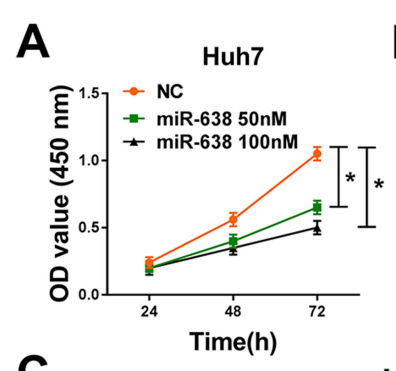

B

Huh7
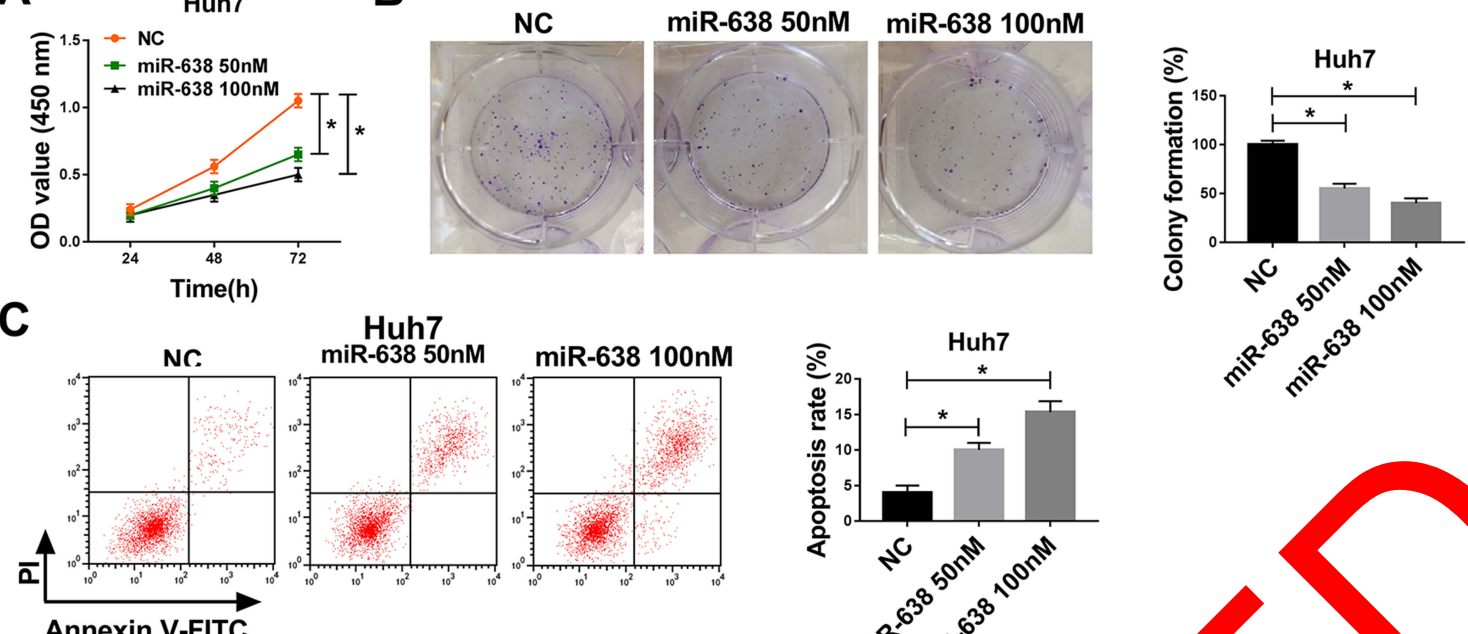

Annexin V-FITC

D

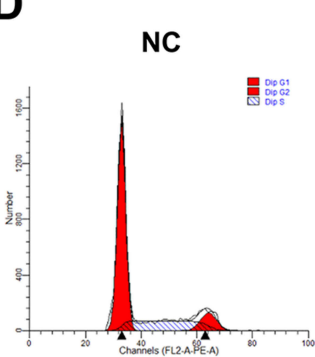

Huh7
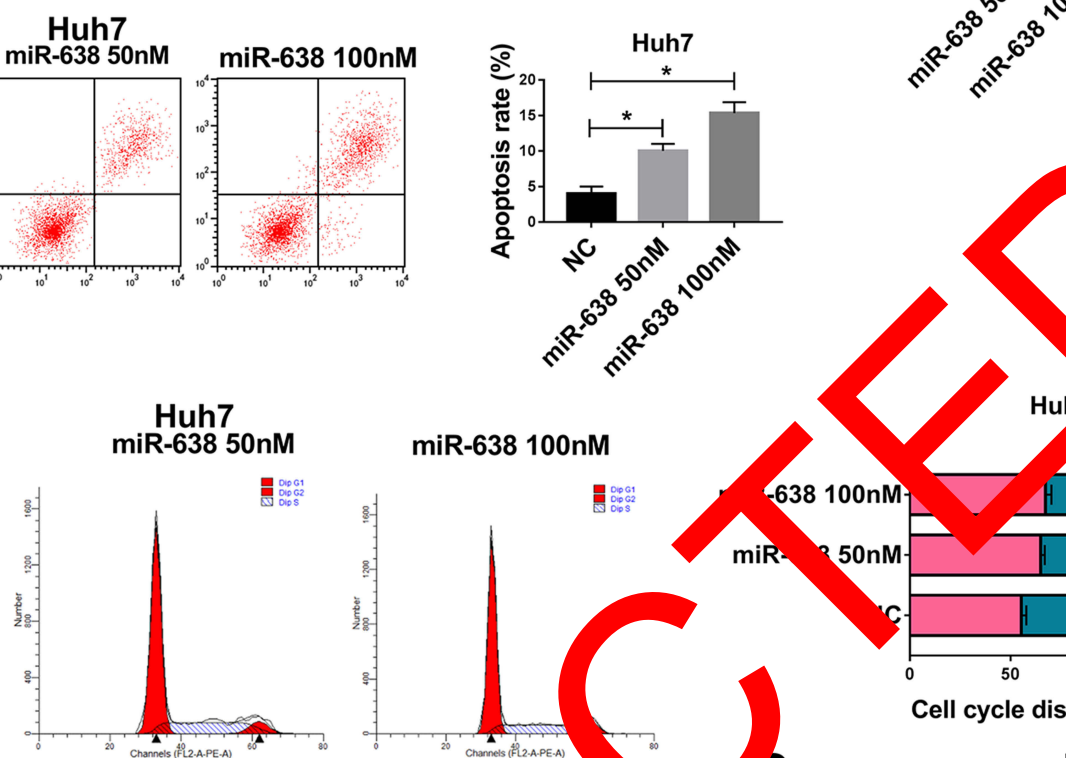

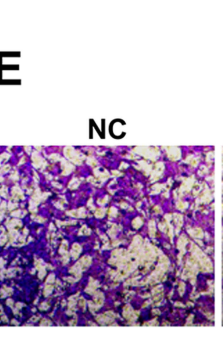

Huh7 miR-638 50nM miR-638 100n

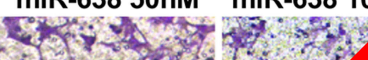
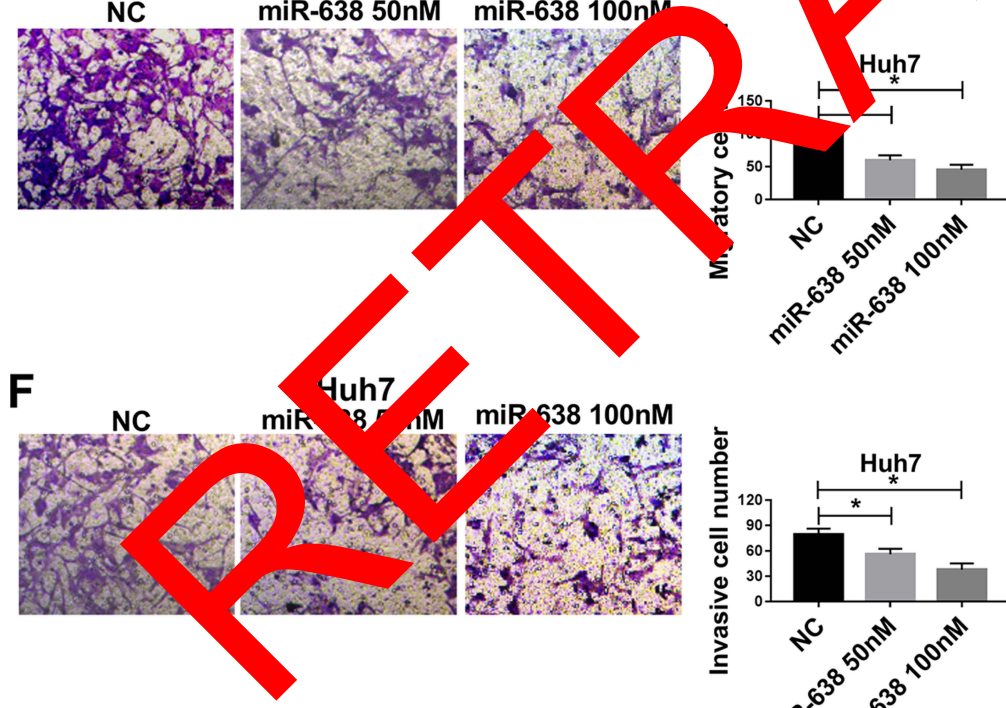

miR-638 100nM
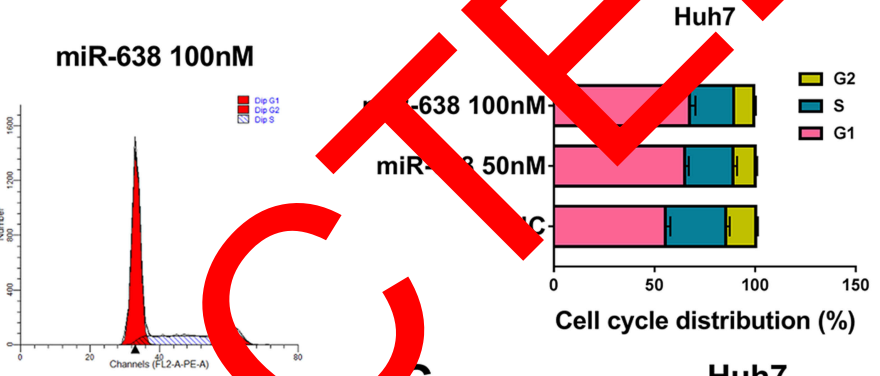

Huh7

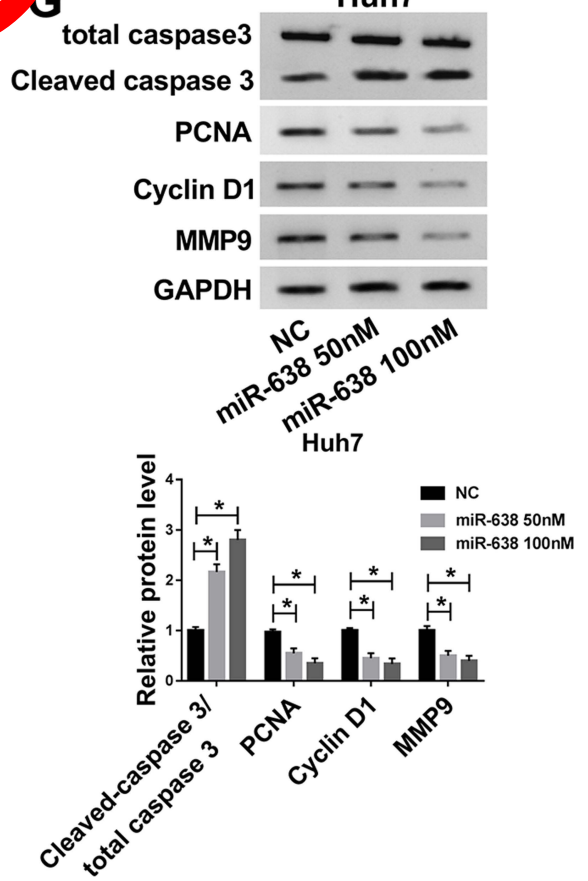

Figure 2 The effect of miR-638 on HCC cell proliferation, migration and invasion. Cell viability (A), colony formation (B), apoptotic rate (C), cell cycle distribution (D), migration $(\mathbf{E})$, invasion $(\mathbf{F})$ and related protein levels $(\mathbf{G})$ were measured in Huh7 cells with transfection of 50 or $100 \mathrm{nM}$ miR-638 mimic or NC via CCK-8, colony formation assay, flow cytometry, transwell and western blot. $* P<0.05$.

the binding sites in SP1-mut group (Figure 4F). Additionally, Ago2 RIP assay showed that SP1 could be bound to miR-638 (Figure 4G). Besides, SP1 protein expression in Huh7 cells was significantly decreased via transfection of miR-638 mimic (Figure 4H). These data suggested that SP1 could be targeted via miR-638 in HCC. 
A

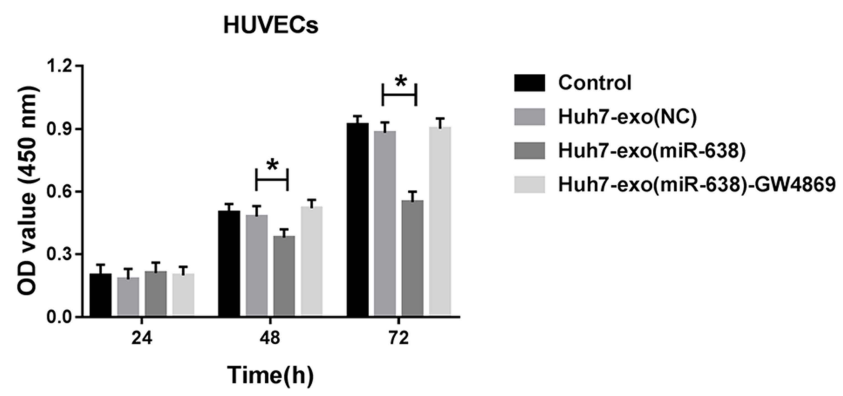

B

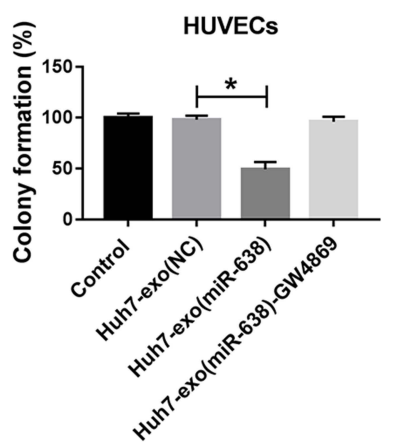

E

HUVECS

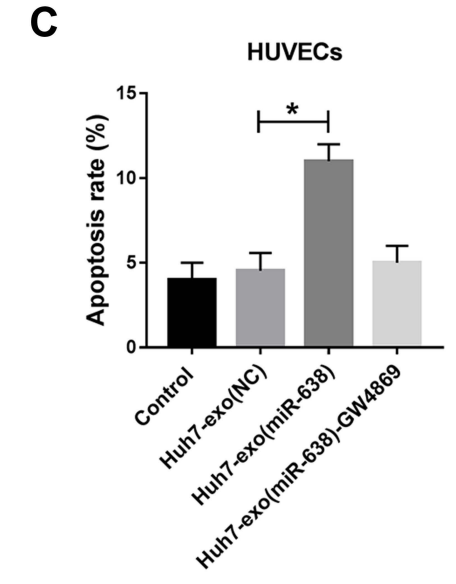

$\mathbf{F}$

HUVECS

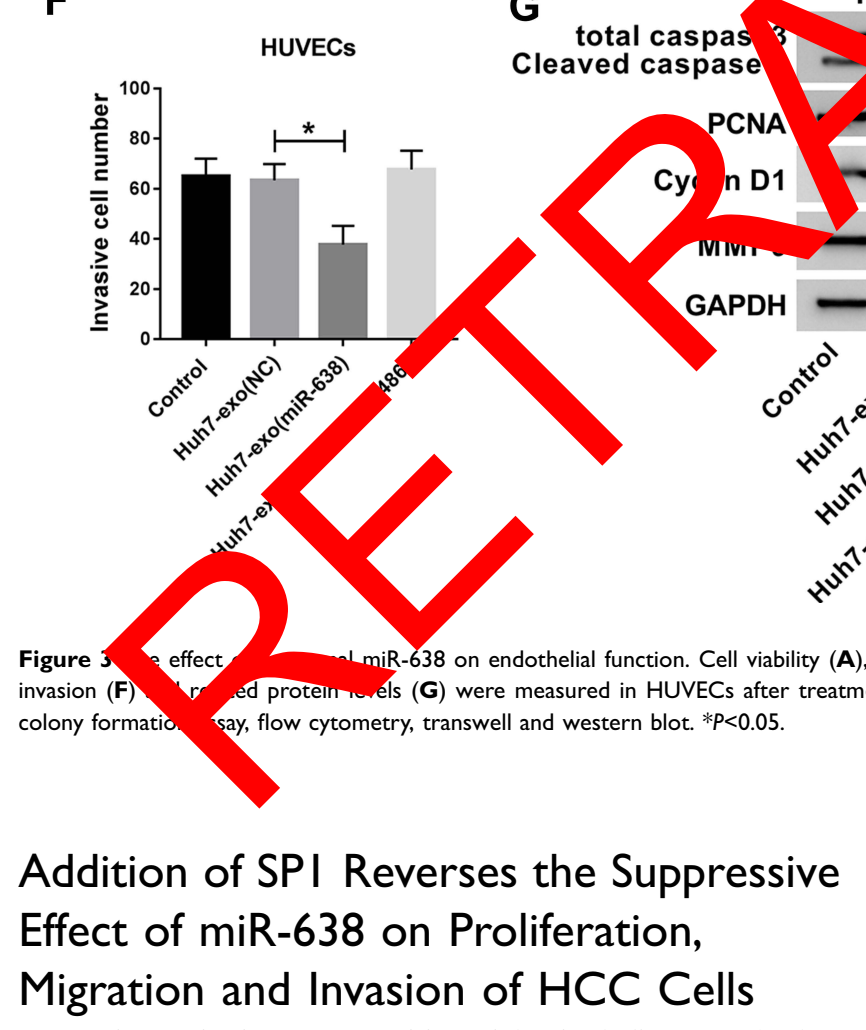

G total caspas
Cleaved caspase HUV

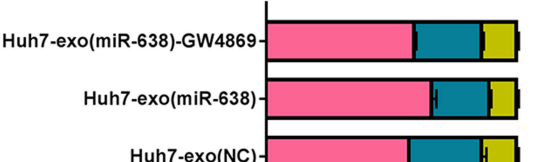

Control

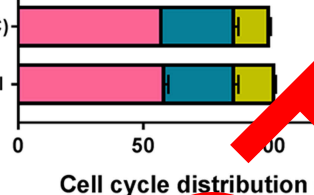

Cell cycle distribution (\%)
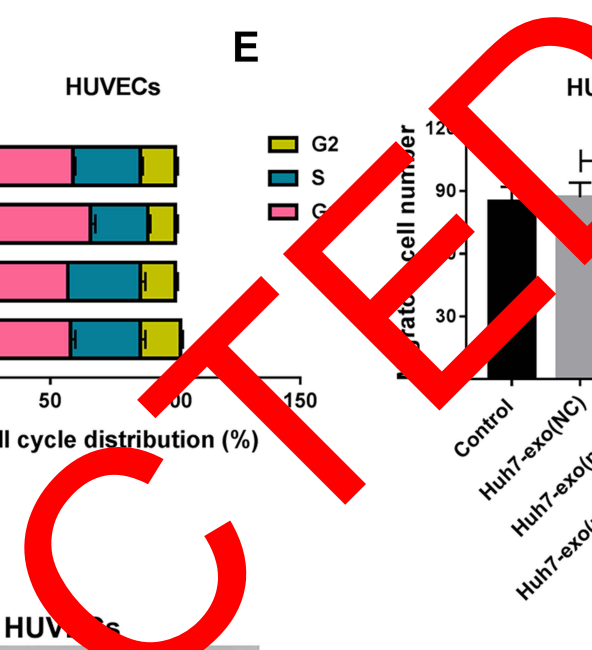

To explore whether SP1 could explain the influence of miR638 in HCC cells, Huh7 cells were transfected with NC, miR638 mimic, miR-638 mimic +vector or SP1 overexpression vector. As exhibited in Figure 5A, introduction of SP1 overexpression vector restored the abundance of SP1 protein in Huh7 cells in the presence of miR-638 mimic. Furthermore, up-regulation of SP1 attenuated miR-638-mediated proliferation reduction by affecting cell viability, colony formation, apoptosis and cell cycle distribution (Figure 5B-E). In addition, restoration of SP1 weakened the inhibitive effect of miR638 on Huh7 cell migration and invasion (Figure 5F and G). 
A
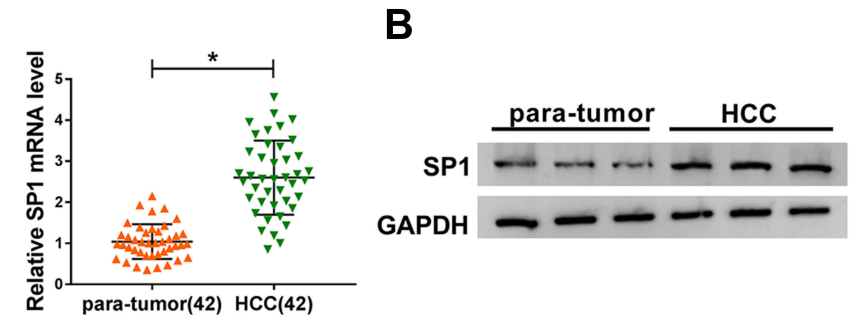

D
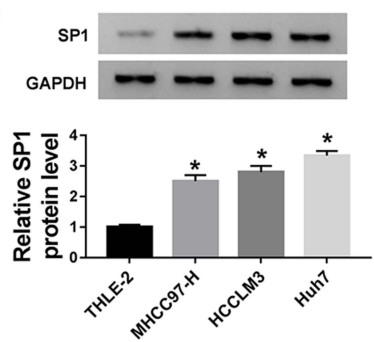

$\mathbf{F}$

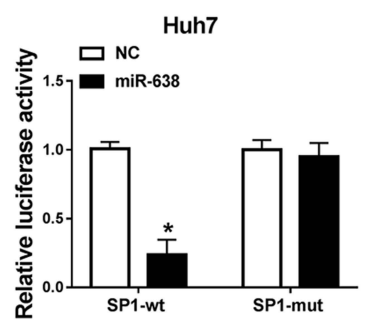

B

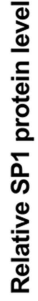

$\mathbf{E}$
G

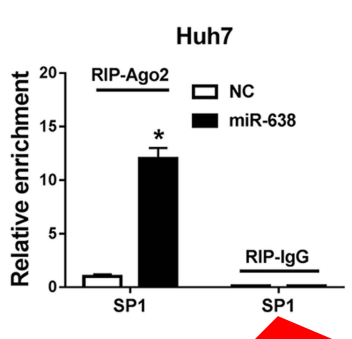

C

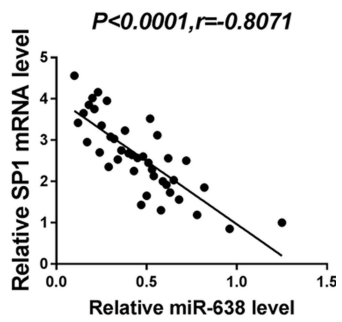

$\mathbf{H}$

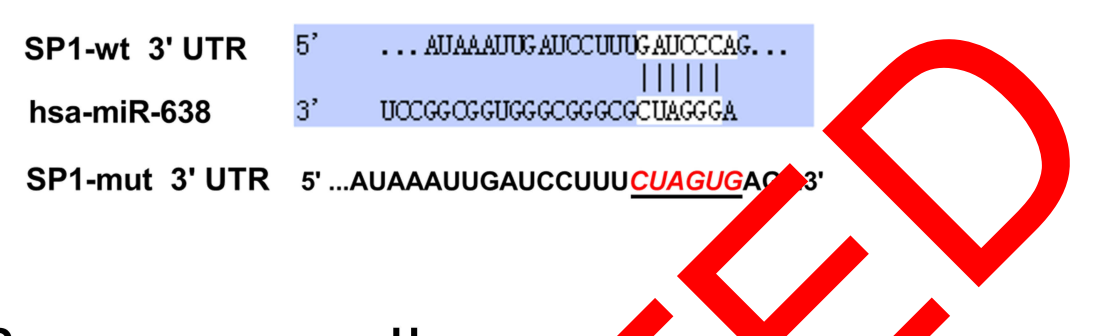

SP1-mut 3' UTR 5'...AUAAAUUGAUCCUUUCUAGUGAC 3'

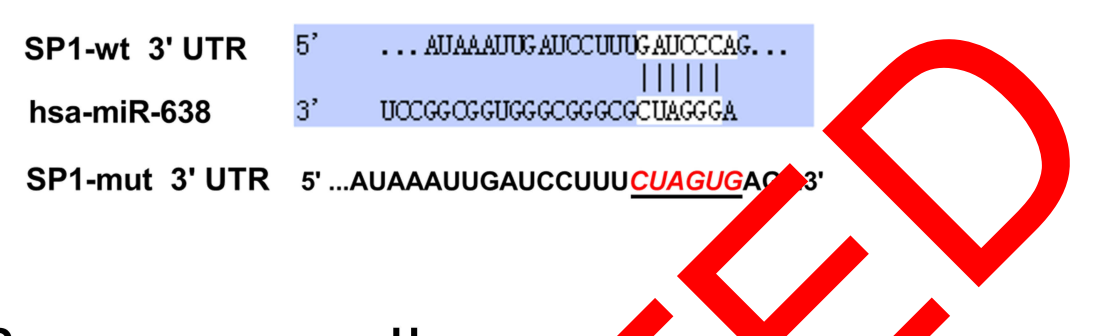

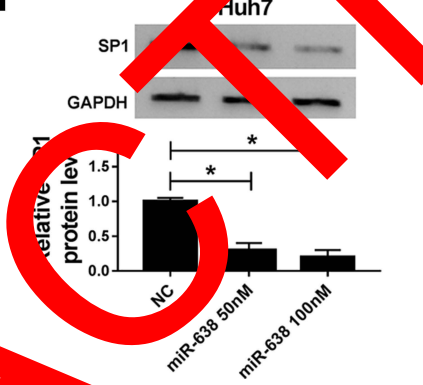

Figure 4 The association between miR-638 and SPI in HCC. (A PPI mRN protein levels in HCC and para-tumor tissues were examined via qRT-PCR and western blot. (C) The linear correlation between expression of $R-638$ d SPI in CC samples was analyzed. (D) SPI protein expression was detected in HCC and normal liver cells. (E) The complementary sequence betwe miR-638 \& SPI wa redicted via starBase. (F and $\mathbf{G})$ Dual-luciferase reporter and RIP assays were conducted in Huh7 cells with transfection of miR-638 mimr NC. (H) ex ression was detected in Huh7 cells transfected with 50 or $100 \mathrm{nM}$ miR-638 mimic or NC. $* P<0.05$.

Besides, overexpression of SP1 evia the effect 0 miR638 on protein levels of Clea a caspase 3, NA, Cyclin D1 and MMP9 in Huh7 cells 1gure 5 $\mathrm{N}$ ). These findings showed that miR-638 repressed $\mathrm{CC}$ proliferation, migration and miR-638 Jer easconenograft Tumor

\section{Growth}

To analyze the role of miR-638 in HCC in vivo, Huh7 cells were used to establish the subcutaneous xenograft model for 9 days, and then tumor sites were treated with miR-638 agomir or NC every other day. As shown in Figure 6A, tumor volume was obviously reduced after the injection of miR-638 agomir for 5, 7 and 10 days. Moreover, the tumor tissues were collected at the ending point. Tumor weight was markedly decreased by treatment of miR-638 agomir (Figure 6B). In addition, miR-638 expression and Cleaved caspase 3 protein level were significantly increased, and protein levels of SP1 and PCNA were remarkably decreased by treatment of miR-638 agomir (Figure 6C and D). These findings suggested that miR638 overexpression decreased HCC development in vivo. A pathway illustration of miR-638/SP1 in HCC development is exhibited in Figure 7.

\section{Discussion}

$\mathrm{HCC}$ is a common cancer with high incidence and mortality, and the effective treatment options for HCC are limited. ${ }^{27}$ Exosomes play important roles in the pathogenesis, diagnosis, development and treatment of $\mathrm{HCC}^{28}$ Moreover, exosomal miRNAs are implicated in the anticancer treatment of HCC. ${ }^{29}$ In our research, the exosomal miR-638 level was reduced in HCC, and exosomal miR638 inhibited HCC cell proliferation, migration and 
A
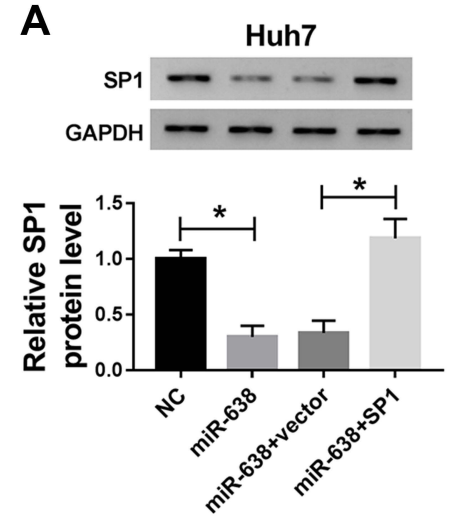

D

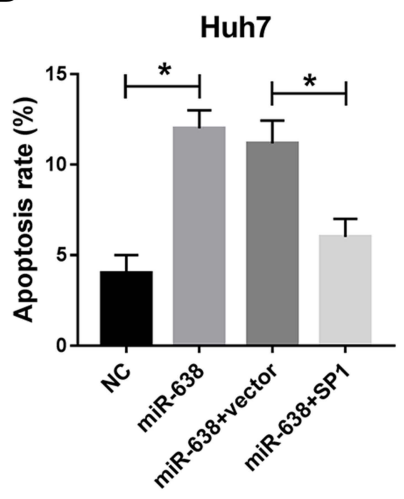

B

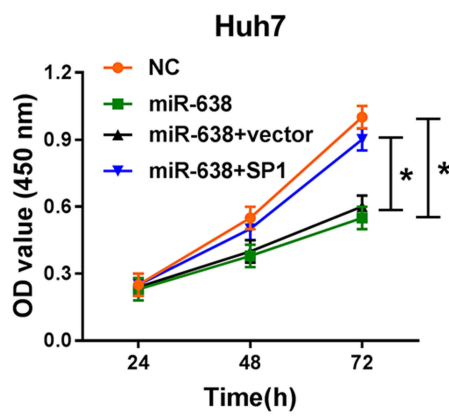

C

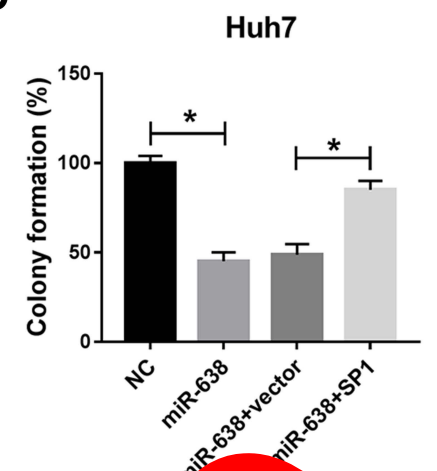

$\mathbf{E}$

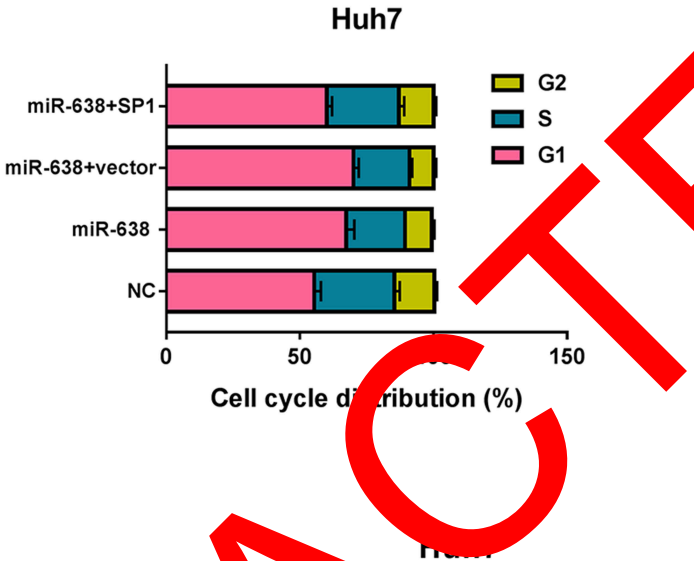

F

G

Huh7

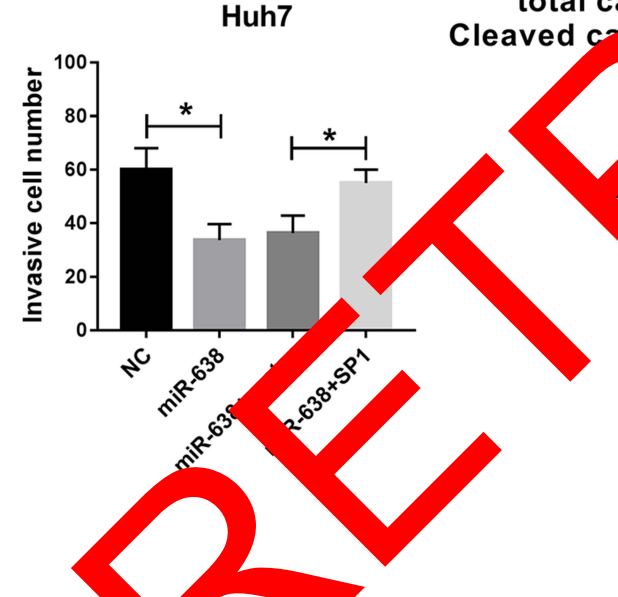

total caspase 3
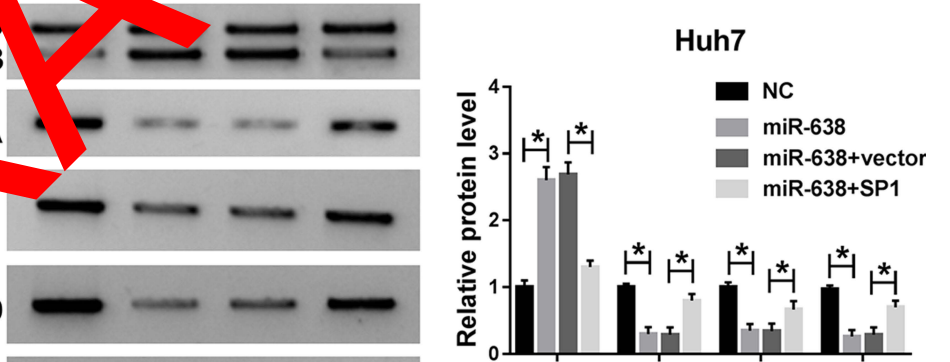

yclin D1 $-\cdots$

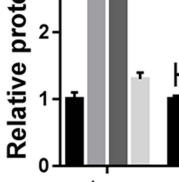

GAPDH
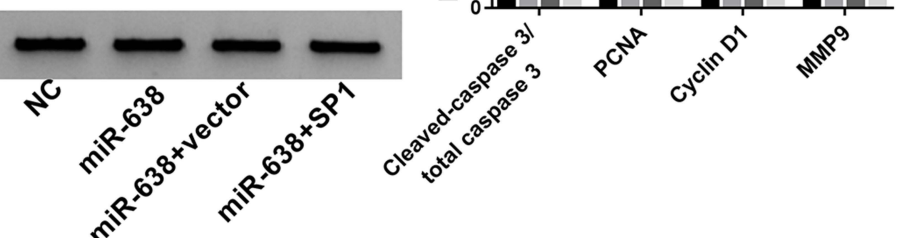

Figure 5 The of miR-638 and SPI on HCC cell proliferation, apoptosis, migration and invasion. SPI protein expression (A), cell viability (B), colony formation (C), apoptotic rate (L. Il cycle distribution $(\mathbf{E})$, migration $(\mathbf{F})$, invasion $(\mathbf{G})$ and related protein abundances $(\mathbf{H})$ were examined in Huh7 cells transfected with NC, I00 $\mathrm{nM}$ miR-638 mimic, miR mimic +vector or SPI. $* P<0.05$.

invasion. Furthermore, here we were the first to confirm the target relationship of miR-638 and SP1 (Figure 7).

The tetraspanins (CD63 and CD81), heat shock protein HSP70 and tumor susceptibility gene TSG101 are the important markers for validating the presence of exosomes. ${ }^{21}$ By detecting these marker levels and TEM, we confirmed that the extracted vesicles were exosomes. Our study displayed that miR-638 abundance was reduced in HCC tissues and cells. We hypothesized the reduced miR-638 might be induced via alteration of the microenvironment. In HCC, the environment might be adverse to miR-638 synthesis or contribute to miR-638 degeneration. A previous study suggested that miR-638 could be released in exosomes from HCC cells. ${ }^{11}$ In this work, exosomal miR-638 was declined 
A

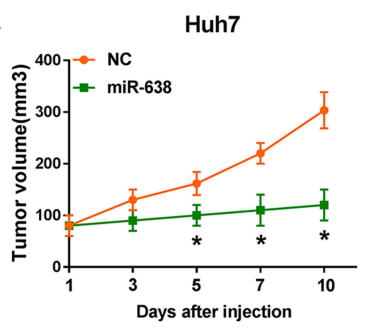

D

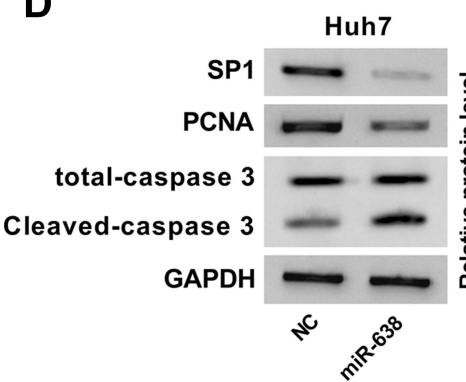

B

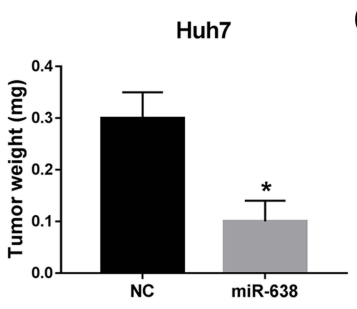

C

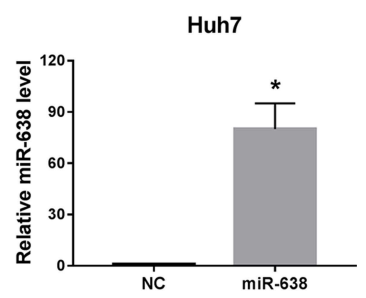

Figure 6 The effect of miR-638 on xenograft tumor growth. $2 \times 10^{6}$ Huh7 cells were subcutaneously injecte nude mice for ay $d$ then mice were treated with miR-638 agomir or NC every other day. $n=5$ per group. (A and B) Tumor volume and weight were me ed in, hroup. (C an, miR-638 expression and protein levels of SPI, PCNA, total caspase 3 and Cleaved caspase 3 were detected in each group. $* P<0.05$.

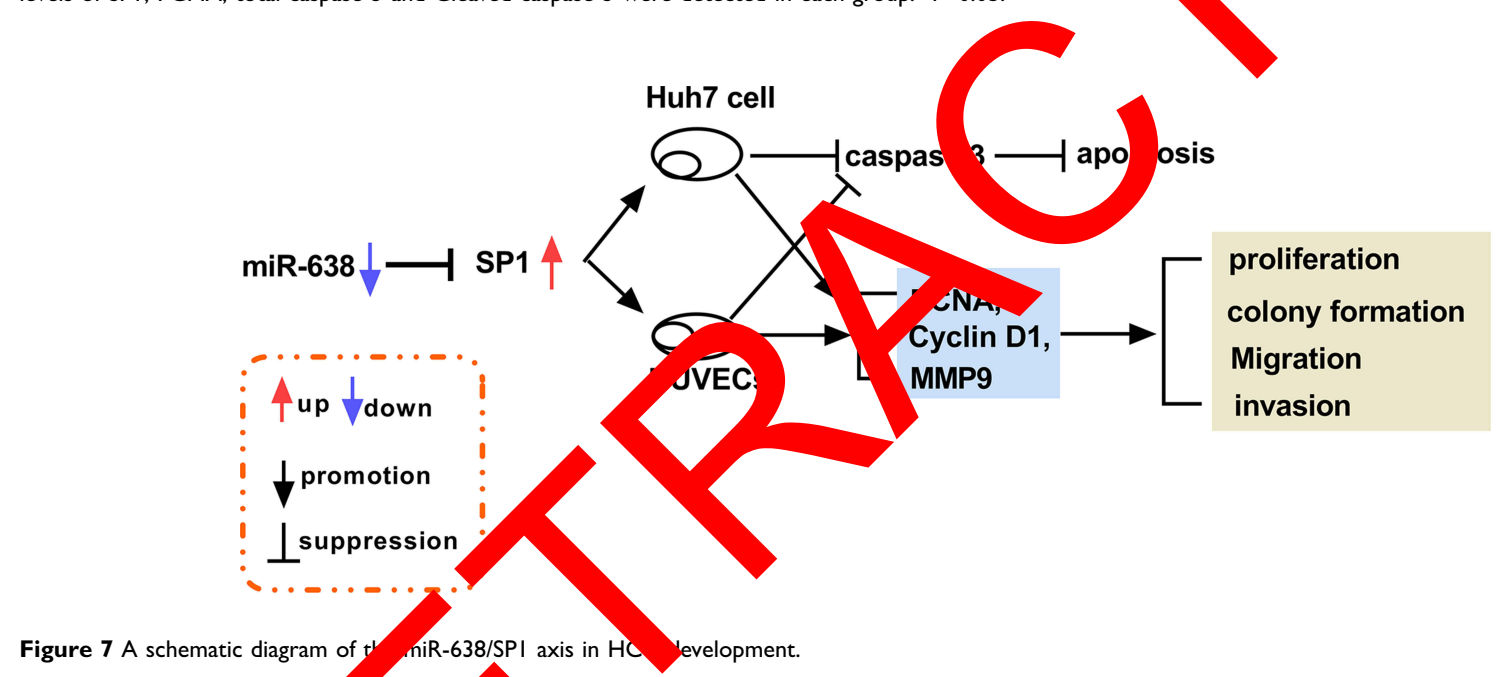

in HCC from sermor ce which as also in agreement with former rk. ${ }^{13}$ eanwh at report indicated that exosomal R-638 in inhibit HCC cell proliferation. ${ }^{13}$ Similarly, we a ound that exosomal miR-638 suppressed HCC cell prolifer. on via restraining cell viability, colony formation, facilitating apoptosis and inducing cell cycle arrest at S1 phase. Moreover, this study showed that miR638 could repress HCC cell migration and invasion, which was also consistent with former work. ${ }^{10}$ In addition, a previous study indicated that inhibition of miR-638 facilitated the angiogenesis in HCC. ${ }^{9}$ Endothelial function is implicated in the angiogenesis, which contributes to tumorigenesis of HCC. ${ }^{30-32}$ Hence, we further assessed the influence of exosomal miR-638 on endothelial function. Using co-culture of Huh7 and HUVECs, we validated that miR-638 from Huh7 cells could be transmitted to HUVECs by exosomes, and then affected HUVEC function. Collectively, this study showed the anti-cancer role of exosomal miR-638 in HCC by inhibiting cell proliferation, migration, invasion and angiogenesis.

miRNAs act as gene regulators by binding the 3'UTR of mRNA to mediate mRNA degradation or translation. ${ }^{33}$ Increasing evidence demonstrated that miR-638 could participate in the regulation of multiple cancers by targeting numerous mRNAs because of the different binding sites, such as phospholipase D1, tetraspanin 1 and sex-determining region Y-box $2 .^{34-36}$ To explore a new mechanism mediated via miR638 , here we were the first to identify that SP1 was targeted via 
miR-638. Previous studies reported that SP1 played an oncogenic role in $\mathrm{HCC}$ via promoting cell proliferation, migration and invasion. ${ }^{16,17,37,38}$ These reports also suggested that miR31-5p, miR-138, miR-363-3p and miR-548b could target SP1 to participate in HCC development, while the expression changes of these miRNAs in exosomes were less than miR638 (Supplementary Figure 2). Furthermore, our study also confirmed the carcinogenic role of SP1 in HCC by rescue experiments. These data also indicated that miR-638 could inhibit HCC development by targeting SP1 in vitro. Besides, the xenograft model is a key tool for understanding the pathogenesis of HCC. ${ }^{39}$ In this research, we also confirmed the anti-cancer role of miR-638 in HCC in vivo using a murine xenograft model. The major function of HUVECs is the ability of vessel formation. Previous studies have reported that SP1 could promote the ability of tube formation of endothelial cells. Thus, we hypothesized that miR-638 might inhibit tube formation of HUVECs via targeting SP1. This would be explored in the future. ${ }^{40,41}$ Although our study demonstrated the function and mechanism of exosomal miR-638 in HCC using Huh7 cells, more cell lines should be used for the study in the future due to the difference of different cell lines.

In conclusion, exosomal miR-638 played an anticancer role in $\mathrm{HCC}$ in vitro and in vivo, possik targeting SP1. This study elucidated a novel mechà ism underlying the pathogenesis of $\mathrm{HCC}$ and dicated potential significance of miR-638 in tre nent $\mathrm{HCC}$.

\section{Funding}

This work was supported b a rrtment of jence and Technology of Liaonino Provina Jinzhou Medical University Joint Fun roject (Grant 20170540336); Natural Science F andation of Liaoning Province (Grant No. 20170540364).

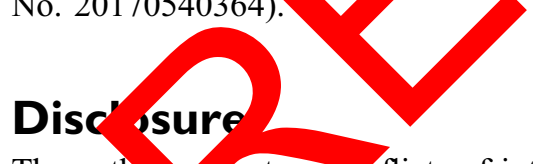

The autho port no conflicts of interest in this work.

\section{References}

1. Llovet JM, Zucman-Rossi J, Pikarsky E, et al. Hepatocellular carcinoma. Nat Rev Dis Primers. 2016;2(1):16018. doi:10.1038/ nrdp. 2016.18

2. Zhu J, Yin T, Xu Y, et al. Therapeutics for advanced hepatocellular carcinoma: recent advances, current dilemma, and future directions. J Cell Physiol. 2019;234(8):12122-12132. doi:10.1002/jcp.28048

3. Wu Q, Zhou L, Lv D, et al. Exosome-mediated communication in the tumor microenvironment contributes to hepatocellular carcinoma development and progression. J Hematol Oncol. 2019;12(1):53. doi:10.1186/s13045-019-0739-0
4. Wang $\mathrm{H}, \mathrm{Lu} \mathrm{Z}$, Zhao $\mathrm{X}$. Tumorigenesis, diagnosis, and therapeutic potential of exosomes in liver cancer. J Hematol Oncol. 2019;12:133. doi:10.1186/s13045-019-0806-6

5. Li C, Xu X. Biological functions and clinical applications of exosomal non-coding RNAs in hepatocellular carcinoma. Cell Mol Life Sci. 2019;76(21):4203-4219. doi:10.1007/s00018-019-03215-0

6. Li S, Yao J, Xie M, et al. Exosomal miRNAs in hepatocellular carcinoma development and clinical responses. J Hematol Oncol. 2018;11(1):54. doi:10.1186/s13045-018-0579-3

7. Cheng J, Chen Y, Zhao P, et al. Dysregulation of miR-638 in hepatocellular carcinoma and its clinical significance. Oncol Lett. 2017;13:3859-3865. doi:10.3892/ol.2017.5882

8. Ye W, Li J, Fang G, et al. Expression of microRNA 638 and sex-determining region Y-box 2 in hepatocellular carcinoma: association between clinicopathological featy prognosis. Oncol Lett. 2018;15:7255-7264. doi:10.3892/

9. Cheng J, Chen Y, Zhao P, et Downregula of miRNA-638 promotes angiogenesis and grom of hepatocell ar carcinoma by targeting VEGF. Oncot et. 201 (21):3070 0711. doi:10.18 632/oncotarget.8930

10. Zhang Y, Zhang D ang J, et Coss of h 038 promotes invasion and epithelial-mese rma ansition by targeting SOX2 in hepatocellular carc ma. On Rep. 20 o7(1):323-332. doi:10.3892/ or.2016.5

11. Kubot o, ba M, Wata M, et al. Secretion of small/ microkNAs inc ing miR-638 into extracellular spaces by sphingophosphod erase 3. Oncol Rep. 2015;33(1):67-73. doi:10.3892/or.2014.3 j

2. Yan S, Dang G, Zhang X, et al. Downregulation of circulating exosomal $\mathrm{m}-638$ predicts poor prognosis in colon cancer patients. Oncotarget. 2017;8(42):72220-72226. doi:10.18632/oncotarget.19 29

13. Shr, slang Y, Yang L, et al. Decreased levels of serum exosomal miR-638 predict poor prognosis in hepatocellular carcinoma. J Cell Duschem. 2018;119(6):4711-4716. doi:10.1002/jcb.26650

14. Giordano S, Columbano A. MicroRNAs: new tools for diagnosis, prognosis, and therapy in hepatocellular carcinoma? Hepatology. 2013;57(2):840-847. doi:10.1002/hep.26095

15. Beishline K, Azizkhan-Clifford J. Sp1 and the 'hallmarks of cancer'. FEBS J. 2015;282:224-258. doi:10.1111/febs.13148

16. Zhao G, Han $C$, Zhang $Z$, et al. Increased expression of microRNA-31-5p inhibits cell proliferation, migration, and invasion via regulating Sp1 transcription factor in HepG2 hepatocellular carcinoma cell line. Biochem Biophys Res Commun. 2017;490 (2):371-377. doi:10.1016/j.bbrc.2017.06.050

17. Liu C, Zhu J, Liu F, et al. MicroRNA-138 targets SP1 to inhibit the proliferation, migration and invasion of hepatocellular carcinoma cells. Oncol Lett. 2018;15(1):1279-1286. doi:10.3892/ol.2017.7357

18. Zou Y, Xiong H, Xiong H, et al. A polysaccharide from mushroom huaier retards human hepatocellular carcinoma growth, angiogenesis, and metastasis in nude mice. Tumour Biol. 2015;36(4):2929-2936. doi:10.1007/s13277-014-2923-8

19. Yu B, Du Q, Li H, et al. Diagnostic potential of serum exosomal colorectal neoplasia differentially expressed long non-coding RNA (CRNDE-p) and microRNA-217 expression in colorectal carcinoma. Oncotarget. 2017;8(48):83745-83753. doi:10.18632/oncotarget.19 407

20. Zheng R, Du M, Wang X, et al. Exosome-transmitted long noncoding RNA PTENP1 suppresses bladder cancer progression. Mol Cancer. 2018;17(1):143. doi:10.1186/s12943-018-0880-3

21. Ekstrom K, Omar O, Graneli C, et al. Monocyte exosomes stimulate the osteogenic gene expression of mesenchymal stem cells. PLoS One. 2013;8(9):e75227. doi:10.1371/journal.pone.0075227

22. Livak KJ, Schmittgen TD. Analysis of relative gene expression data using real-time quantitative PCR and the $2-\Delta \Delta C$ T method. Methods. 2001;25(4):402-408. doi:10.1006/meth.2001.1262 
23. Wang SC. PCNA: a silent housekeeper or a potential therapeutic target? Trends Pharmacol Sci. 2014;35:178-186. doi:10.1016/j. tips.2014.02.004

24. Qie S, Diehl JA. Cyclin D1, cancer progression, and opportunities in cancer treatment. J Mol Med (Berl). 2016;94(12):1313-1326. doi:10.1007/s00109-016-1475-3

25. Nagata S. Apoptosis and clearance of apoptotic cells. Anпu Rev Immunol. 2018;36(1):489-517. doi:10.1146/annurev-immunol-042617-053010

26. Huang H. Matrix metalloproteinase-9 (MMP-9) as a cancer biomarker and MMP-9 biosensors: recent advances. Sensors (Basel). 2018;18(10):3249. doi:10.3390/s18103249

27. Bupathi M, Kaseb A, Meric-Bernstam F, et al. Hepatocellular carcinoma: where there is unmet need. Mol Oncol. 2015;9(8):1501-1509. doi:10.1016/j.molonc.2015.06.005

28. Moris D, Beal EW, Chakedis J, et al. Role of exosomes in treatment of hepatocellular carcinoma. Surg Oncol. 2017;26(3):219-228. doi:10.1016/j.suronc.2017.04.005

29. Pan J-H, Zhou H, Zhao -X-X, et al. Role of exosomes and exosomal microRNAs in hepatocellular carcinoma: potential in diagnosis and antitumour treatments (Review). Int $J$ Mol Med. 2018;41 (4):1809-1816. doi:10.3892/ijmm.2018.3383

30. Liu P, Atkinson SJ, Akbareian SE, et al. Sulforaphane exerts anti-angiogenesis effects against hepatocellular carcinoma through inhibition of STAT3/HIF-1alpha/VEGF signalling. Sci Rep. 2017;7:12651. doi:10.1038/s41598-017-12855-w

31. Morse MA, Sun W, Kim R, et al. The role of angiogenesis in hepatocellular carcinoma. Clin Cancer Res. 2019;25(3):912-920. doi:10.1158/1078-0432.CCR-18-1254

32. Zhao S, Li J, Zhang G, et al. Exosomal miR-451a functions as a tumor suppressor in hepatocellular carcinoma by targeting LPIN1. Cell Physiol Biochem. 2019;53:19-35. doi:10.33594/000000118
33. Li D, Zhang J, Li J. Role of miRNA sponges in hepatocellular carcinoma. Clin Chim Acta. 2020;500:10-19. doi:10.1016/j.cca.2019.09.013

34. Tang KL, Tang HY, Du Y, et al. MiR-638 suppresses the progression of oral squamous cell carcinoma through wnt/beta-catenin pathway by targeting phospholipase D1. Artif Cells Nanomed Biotechnol. 2019;47:3278-3285. doi:10.1080/21691401.2019.1647222

35. Zhang J, Fei B, Wang Q, et al. MicroRNA-638 inhibits cell proliferation, invasion and regulates cell cycle by targeting tetraspanin 1 in human colorectal carcinoma. Oncotarget. 2014;5(23):12083-12096. doi:10.18632/oncotarget.2499

36. Xia Y, Wu Y, Liu B, et al. Downregulation of miR-638 promotes invasion and proliferation by regulating SOX2 and induces EMT in NSCLC. FEBS Lett. 2014;588(14):2238-2245. doi:10.1016/j.febslet.2014.05.002

37. Ying J, Yu X, Ma C, et al. MicroRNA-363-3p is downregulated in hepatocellular carcinoma and inhibits tum is by directly targeting specificity protein 1. Mol Med p. 2017, 2):1603-1611. doi: $10.3892 / \mathrm{mmr} 2017.6759$

38. Qiu H, Zhang G, Song B, et al,Micro LA-548b inhi s proliferation and invasion of hepatocel' ar carcino cells by ectly targeting specificity protein 1 Exp Ther Mev 20 18:2332-2340. doi: $10.3892 /$ etm. 2019.7

39. Fornari F, Gramantier ${ }^{-1}$, car et al. Micr RNAs in animal models of HCC. Cancers (sel). 201 doi:10.3 d/cancers 11121906

40. Xie J, Gong iu X, et al. ans ption factor SP1 mediates hyperglyce ind re upregulatio roundabout4 in retinal microvascular endothelial 1s. Gene. 2017;616:31-40. doi:10.1016/j. gene 3.027

41. Jj g Y, Liu H, Liu WJ, $M$ al. Endothelial Aquaporin-1 (AQP1) pression is re lated by transcription factor Mef2c. Mol Cells. 16;39(4):292-2 doi:10.14348/molcells.2016.2223

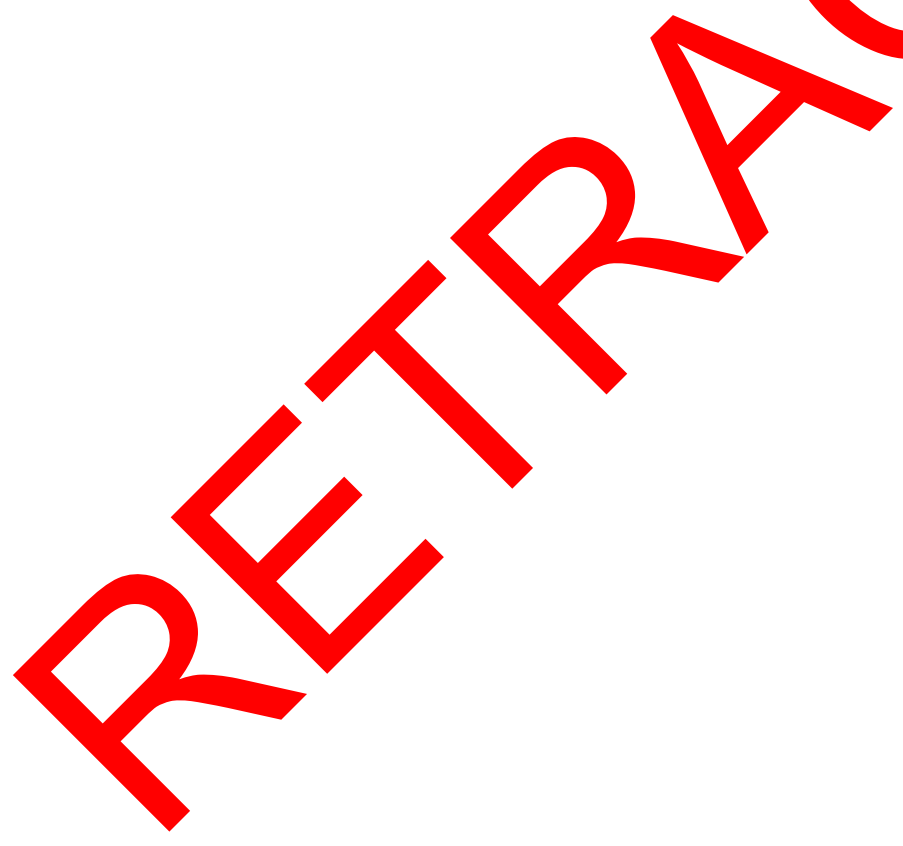

OncoTargets and Therapy

Dovepress

\section{Publish your work in this journal}

OncoTargets and Therapy is an international, peer-reviewed, open access journal focusing on the pathological basis of all cancers, potential targets for therapy and treatment protocols employed to improve the management of cancer patients. The journal also focuses on the impact of management programs and new therapeutic

Submit your manuscript here: https://www.dovepress.com/oncotargets-and-therapy-journal agents and protocols on patient perspectives such as quality of life, adherence and satisfaction. The manuscript management system is completely online and includes a very quick and fair peer-review system, which is all easy to use. Visit http://www.dovepress.com/ testimonials.php to read real quotes from published authors. 